\title{
Simultaneous atrial and ventricular pacing during narrow complex tachycardia - The Janus Response. Which one is true?
}

Anand Manickavasagam ${ }^{1}$, Sirish Chandra Srinath Patloori ${ }^{1}$, Hariharan Narasaiyan ${ }^{2}$, David Chase $^{2}$, and John Jacob ${ }^{3}$

${ }^{1}$ Christian Medical College and Hospital Vellore

${ }^{2}$ Christian Medical College Vellore

${ }^{3}$ Christian Medical College

September 25, 2021

\section{Title Page Manuscript Title:}

Simultaneous atrial and ventricular pacing during narrow complex tachycardia - The Janus Response. Which one is true?

\section{Short Running title:}

Simultaneous A and V pacing - Janus responseKeywords: Atypical AVNRT, lower common pathway, VA linking, ventricular overdrive pacing

\section{Authors details:}

1. Dr. Anand Manickavasagam, MBBS, MD, DM, Department of Cardiology, Christian Medical College, Vellore, Tamilnadu, India

2. Dr. Sirish Chandra Srinath Patloori, MBBS, MD, DM, CEPS, Department of Cardiology, Christian Medical College, Vellore, Tamilnadu, India

3. Dr. Hariharan Narasaiyan, MBBS, MD, DM, Department of Cardiology, Christian Medical College, Vellore, Tamilnadu, India

4. Dr. David Chase, MBBS, MD, DM, Professor, Department of Cardiology, Christian Medical College, Vellore, Tamilnadu, India

5. Dr. John Roshan, MBBS, MD, DM, CEPS, CCDS, Professor, Department of Cardiology, Christian Medical College, Vellore, Tamilnadu, India

\section{Corresponding Author:}

Dr. John Roshan, Cardiology Unit IV, Department of Cardiology, Christian Medical College, Vellore, Tamilnadu, India, 632004 Phone: 91-04162283572, 91-9443312680 E-mail id:johnroshanjacob@gmail.comAcknowledgments: NoneFinancial disclosure: None for all the authors No conflict of interestEthical Statement: As this was a case report ethical clearance was not 
obtained. The patient's guardian consent was obtained before the procedure towards the publication of the anonymized clinical details if found suitable for a medical journal. Hence, we request you to waive off the ethical committee clearance.Data availability Statement: The data that supports the finding of the study are available on request from the corresponding author.

\section{Funding Statement: None}

Case PresentationA 13-year-old boy presented with recurrent episodes of palpitations and a normal baseline electrocardiogram (ECG). He underwent a cardiac electrophysiology study with the intent of radiofrequency ablation. Four diagnostic electrophysiology catheters were placed - a decapolar catheter in the coronary sinus (CS) and three other quadripolar catheters placed at AV junction in the 'His bundle' region, right ventricular (RV) apex, and right atrium (RA). Baseline intervals were normal. A narrow QRS tachycardia (Tachycardia 1) was induced during catheter placement with a cycle length of $300 \mathrm{~ms}$ and AH longer than HA interval (Figure 1a). The earliest atrial activation was in the proximal CS electrodes. Ventricular pacing at a cycle length of $280 \mathrm{~ms}$ showed ventricular capture without reset of the atrium or change in its activation sequence. However, on cessation of ventricular pacing, a different narrow QRS tachycardia (Tachycardia 2) was seen at a cycle length of $290 \mathrm{~ms}$ with AH interval shorter than HA interval, and VA interval of $180 \mathrm{~ms}$ (Figure 1b). Differential atrial overdrive pacing from three different sites showed a maximum $\Delta \mathrm{V}$-A interval of the first return beats post-cessation of pacing to be less than $10 \mathrm{~ms}$ for tachycardia 1 . This manoeuvre could not be utilised for tachycardia 2 as the tachycardia got terminated. On performing "simultaneous atrial and ventricular pacing" (RA+RV pacing) at a cycle length of $270 \mathrm{~ms}$ during tachycardia 2 - the first event after cessation of pacing was always an atrial electrogram (Figure 2a). The same manoeuvre when performed for tachycardia 1 showed an atrial EGM as the first event with change to tachycardia 2 (Figure 2b) every time, excepting for one occasion where the initial event was a His bundle electrogram (HBE) with tachycardia 1 which continued (Figure 2c). How do we electrophysiologically reconcile the Janus or duality of responses with - RA+RV pacing during tachycardia 1 and what is the likely mechanism for the two tachycardias?

\section{Discussion:}

Tachycardia 1 had the earliest atrial activation in proximal CS and the AH interval was longer than the HA interval. Attempt to entrain from the RV apex, dissociated the ventricle without tachycardia termination. However, after cessation of ventricular over-drive pacing (VOP) despite changes in $\mathrm{AH}$ and HA intervals, the atrial activation pattern remained the same with the earliest atrial EGM in the proximal CS - as shown in tachycardia 2. The presence of AV dissociation ruled out orthodromic Atrio- Ventricular Reentrant tachycardia (o-AVRT) leaving us with two alternative diagnoses - (1) atrial tachycardia (AT) with origin near the proximal CS with conduction via slow AV nodal pathway in tachycardia 1 and via fast AV nodal pathway in tachycardia 2, or (2) both the tachycardias being various forms of atrioventricular nodal reentrant tachycardia (AVNRT). ${ }^{1}$ Probably tachycardia 1 was a slow/slow form and tachycardia 2 was a fast/slow form of AVNRT. Differential atrial overdrive pacing showed "VA linking", suggesting tachycardia 1 was AVNRT. ${ }^{2}$ This manoeuvre could not be done during tachycardia 2 due to repeated termination. However, tachycardia 2 had identical atrial activation to tachycardia 1 and was reproducibly induced with ventricular pacing, making atrial tachycardia very unlikely. This implied that both the tachycardias were likely different forms of atypical AVNRT. In situations where a narrow complex tachycardia cannot be entrained by VOP, besides demonstrating VA linking by differential site atrial pacing, RA + RV pacing proposed by Saba et.al can be useful to differentiate between AT and AVNRT. ${ }^{3}$ If the first event after cessation of RA + RV pacing during narrow complex tachycardia is a His electrogram it suggests AVNRT, while if atrial it suggests AT. Although their study did not include atypical AVNRT, "given the proximity of the His bundle to the tachycardia circuit they suspected that the response to RA + RV pacing would be similar to that of typical AVNRT." ${ }^{3}$ Like the 
Roman God Janus who looks simultaneously in opposite directions we were faced with discordant responses to this manoeuvre. The first event after RA+RV pacing in tachycardia 2 was always atrial (Figure 2a). On most occasions during tachycardia 1, the first event after RA+RV pacing was also an atrial electrogram (Figure 2b). Interestingly, on cessation of pacing tachycardia 1 changed to tachycardia 2 multiple times. Considering the evidence discussed above, both tachycardias were likely to be different forms of AVNRT and in both instances, with RA + RV pacing the His bundle was activated retrogradely (AH during pacing was shorter than tachycardia, and the His morphology was different). In tachycardia 2 (a fast-slow form of AVNRT with a probable lower common pathway), during RA+RV pacing the likely site of a collision between the two opposing wavefronts was in the lower common pathway thereby leaving the tachycardia unaffected (Figure 3a). Consequently, when the pacing was stopped, the tachycardia resumed, and the first event was an atrial electrogram. In tachycardia 1 (a slow-slow form of AVNRT), ventricular pacing likely reached the tachycardia circuit and collision was probably above the lower turn-around point (Figure $3 \mathrm{~b}$ ). On continued pacing, the antegrade conduction likely changed from the slow pathway to the fast pathway due to retrograde concealed conduction from the previous ventricular paced beat into the slow pathway. This resulted in the transition of tachycardia 1 to tachycardia 2 and explains the first event after pacing being the atrial electrogram. (Figure 3b). In our case, only on one occasion during tachycardia 1 , the first event after RA+RV pacing was a His electrogram (Figure 2c). However, on careful analysis, it was observed that the His was activated anterogradely during RA + RV pacing (similar AH interval and same His morphology during tachycardia and pacing). Further evidence in favour of antegrade His activation was the presence of His electrogram coinciding with pacing spike artefact on His channel and ventricular fusion in surface leads when compared to fully paced QRS complex in Figure $2 \mathrm{~b}$. The 2 paced wavefronts collided in the ventricle effectively leaving only the atrial pacing wavefront to interact with the tachycardia circuit. So, technically, this was only atrial pacing and not RA + RV pacing (Figure 3c). The His response should be interpreted with caution as just atrial pacing can result in a similar response in both AT and AVNRT. Radiofrequency energy was delivered at the site of the right inferior extension of the AV node below the level of the roof of the CS ostium. There was accelerated junctional ectopy during the delivery of RF energy. However, both the tachycardias were inducible after this. Then, the earliest atrial activation was mapped to the roof of the proximal CS during tachycardia 2, and ablation was performed at this location during sinus rhythm. Post ablation there was no tachycardia inducible. Even though the presence of a lower common pathway is refuted by many electrophysiologists, we postulated the model employing a lower common pathway for easy understanding. It may be a lower common pathway or "longer refractory period below the circuit" as suggested by D.G. Katritsis and M.E. Josephson. ${ }^{1}$ This case highlights that the first intracardiac electrical signal that follows RA+ RV pacing for an atypical fast-slow form of AVNRT can be atrial. Hence, this manoeuvre is unlikely to help distinguish atypical forms of AVNRT from AT. Attention should also be paid to the activation of the His bundle to make correct interpretations.

\section{References:}

1. Katritsis DG, Josephson ME. Classification of electrophysiological types of atrioventricular nodal reentrant tachycardia: a reappraisal. Europace. 2013 Sep;15(9):1231-40.

2. Maruyama M, Kobayashi Y, Miyauchi Y, Ino T, Atarashi H, Katoh T, et al. The VA relationship after differential atrial overdrive pacing: a novel tool for the diagnosis of atrial tachycardia in the electrophysiologic laboratory. J Cardiovasc Electrophysiol. 2007 Nov;18(11):1127-33.

3. Saba S, Bhattacharya S, Mezu U, Razak E, Mendenhall GS, Adelstein E, et al. A novel manoeuvre for discerning supraventricular tachycardia mechanism. Europace. 2011 Apr 1;13(4):562-5. 


\section{Figure legends:}

Figure 1: Intracardiac electrogram with intervals of tachycardia 1 in Figure 1a and tachycardia 2 in Figure 1b. From top to bottom, I, aVF, V1 represents; leads I, avF, and V1 of 12 lead ECG. RA d represents the distal bipole of the right atrial catheter. His $\mathrm{p}$ and His d represent proximal to distal poles of His bundle catheter. CS 9-10 to 1-2 represent proximal to distal poles of a decapolar catheter in the coronary sinus. RV $d$ represents the distal bipole of the catheter in the right ventricular apex. Figure 2: Simultaneous atrial and ventricular pacing from proximal CS and RV apex, respectively. Figure 2a shows the initial atrial electrogram after cessation of pacing for tachycardia 2 . Figure $2 \mathrm{~b}$ shows the initial atrial electrogram after cessation of pacing for tachycardia 1. Figure 2c shows initial His electrogram after cessation of pacing for tachycardia 1. Catheter position and abbreviation same as in Figure 1. H represents His bundle electrogram. RA + RV - simultaneous RA and RV pacing. Figure 3: Ladder diagram explaining the probable mechanism for the response to simultaneous atrial and ventricular pacing. A - Atrium, H - His bundle, LCP - lower common pathway, V - Ventricle, \#- refractoriness, green star - pacing, green circle - collision, and dotted lines represent continued A and V pacing for few beats before the last paced beat.
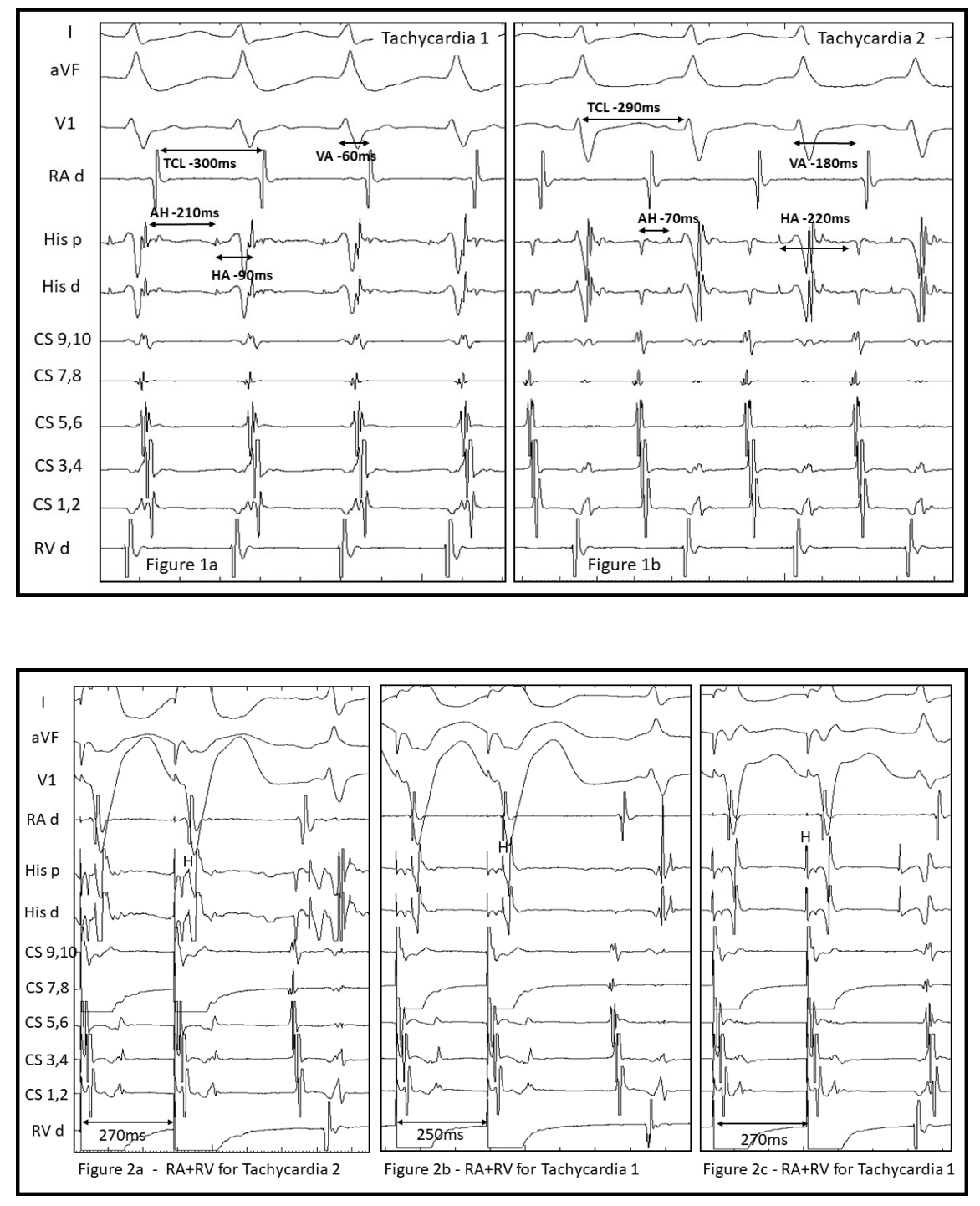

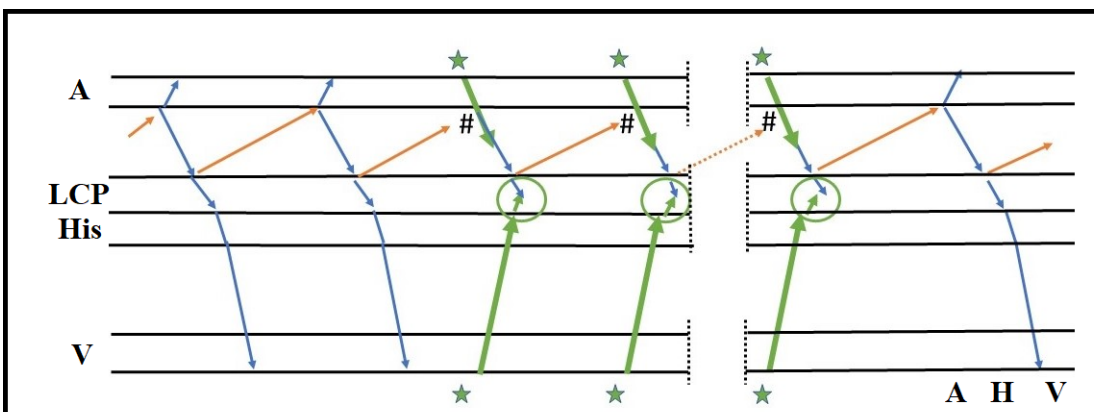

Figure 3a

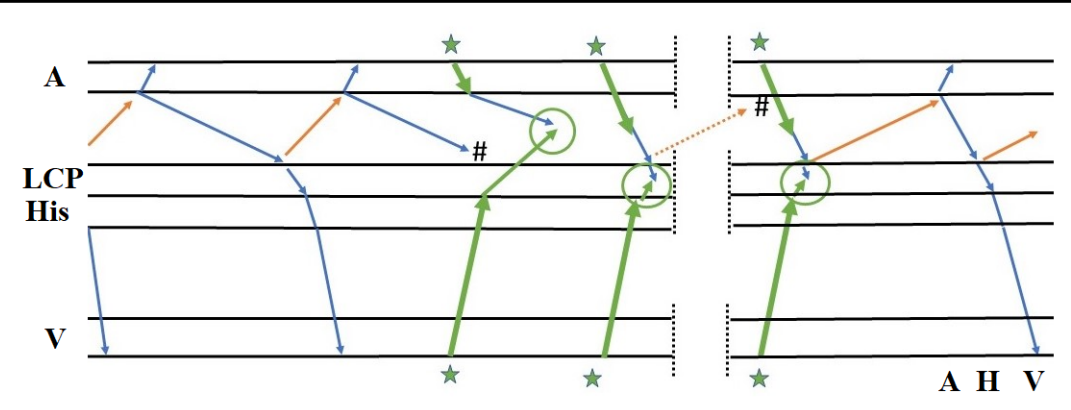

Figure 3b

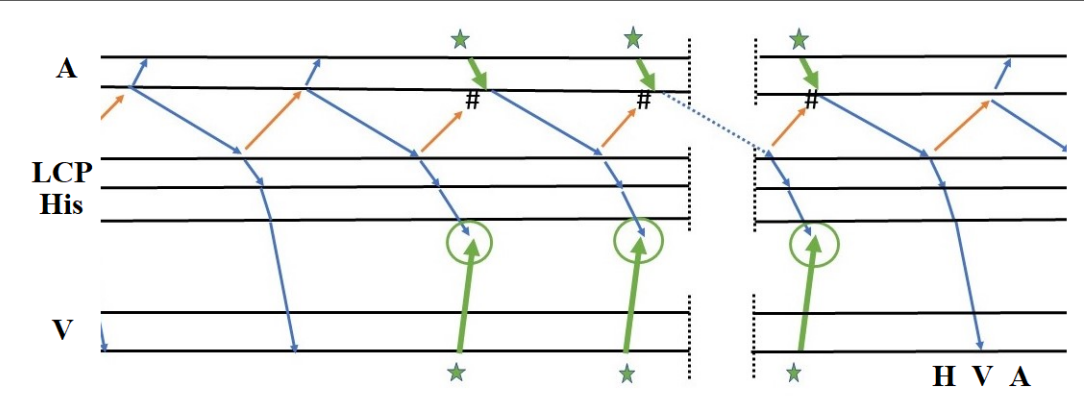

Figure 3c 